\title{
Bio-Évaluation De La Qualité Des Eaux De Surface D'oued Beht (Maroc) Indice Biologique Global Des Réseaux De Contrôle Et De Surveillance (IBG-RCS)
}

\author{
Lamhasni Nihad \\ Chillasse Lahcen \\ Timallouka Mohamed
}

Laboratoire de l'équipe d'écologie et biodiversité des zones humides.

Dép. Biologie Zitoune Meknès.

Université Moulay Ismail, Faculté des Sciences Meknès, Maroc

doi: 10.19044/esj.2016.v13n3p94 URL:http://dx.doi.org/10.19044/esj.2016.v13n3p94

\begin{abstract}
The present study concerns the biological assessment of Beht's river quality in Morocco during the period Mai-June of 2014 using the standard Global Biological Index of Control and Surveillance Networks (GBICSN).Thus twelve sampling stations were chosen for the determination of the impact of agricultural and urban waste of Beht's area on the biological waters quality of this river. As results this biological approach showed downgrading notes of the biological index from the watershed's uphill to its downstream reflecting this way the deterioration of water quality with increasing anthropogenic action and inputs.
\end{abstract}

Keywords: Beht's river, biological index, Biological quality, macroinvertebrates, anthropogenic inputs

\section{Résumé}

La présente étude a pour but la détermination de la qualité biologique des eaux du bassin versant d'Oued Beht pendant l'année 2014 par l'utilisation de l'Indice Biologique Global des Réseaux de Contrôle et de Surveillance (IBG-RCS) normalisé, indice relatif à l'étude de la macrofaune benthique des eaux douces. Les résultats obtenus au niveau des 12 stations d'échantillonnage choisies pour la détermination de l'impact des différentes activités humaines (rejets agricoles, urbains et industriels) sur la qualité biologique des systèmes lotiques de ce bassin montrent la diminution de la note de l'indice biologique des stations situées sur le Beht amont vers les stations situées sur le Beht aval, laquelle diminution est subséquente à l'effondrement de la richesse en invertébrés dans la zone avale du bassin 
versant. Ces résultats reflètent la dégradation de la qualité du milieu aquatique suite à l'augmentation des apports anthropiques de la région, l'extraction des matériaux alluvionnaires ou encore l'installation des ouvrages hydroélectriques qui perturbent le régime hydrologique des systèmes lotiques.

Mots-clés : Oued Beht, indice biologique, qualité biologique, macroinvertébrés, impact anthropique

\section{Introduction}

Sur la Terre, l'eau destinée à la consommation humaine ne présente qu'une petite portion égale à moins de $1 \%$. Cet élément vital présent dans les rivières et cours d'eau avec un pourcentage de $0,006 \%$ est d'une importance indéniable et obligatoire à la vie de l'écosystème mondial (Malmqvist \& Rundle, 2002). En effet cette ressource répond aux besoins majeurs de l'humanité sur Terre, ses besoins qui résident dans l'utilisation des eaux dans tous les domaines vitaux comme l'agriculture, l'industrie, la pèche, la production de l'énergie, le transport... (Malmqvist and Rundle, 2002; United Nations Environment Programme Global Environnement Monitoring System Water Programme [UNEP (GEMS)/WP], 2008).

Par ailleurs cet élément clé de développement humain se trouve fortement atteint et négativement affecté par l'augmentation accrue de la population humaine au monde entier, également son utilisation anarchique et non durable (Echihabi et al., 2015; Bzioui, 2004) qui touche considérablement non seulement à la qualité des eaux mais cause également un grand déclin de la biodiversité des systèmes lotiques au travers le Monde (Liquete et al., 2011)

La caractérisation de l'état des écosystèmes lotiques, la détection des agressions, la prévention, la surveillance et l'opposition aux transformations indésirables exigent des moyens de diagnostic efficaces et fiables (Blandin, 1986; United States Environmental Protection Agency [U.S.EPA], 1997). L'approche physicochimique dans l'évaluation de la qualité des eaux permet la caractérisation des conditions dans lesquelles fonctionnent les écosystèmes aquatiques au moment des analyses, mais elle ne permet évidemment de tirer des conclusions sur la santé de ces écosystèmes. Pour ce faire, les mesures relatives aux êtres vivants sont indispensables (Blandin, 1986).

L'approche biologique se base sur l'utilisation des indices biotiques qui combinent une mesure quantitative de diversité à une mesure qualitative basée sur la présence ou l'absence des taxons sensibles à la pollution (Hilsenhoff, 1988; Bode et al., 1991). Parmi les indices biotiques, l'Indice Biologique Global des Réseaux de Contrôle et de Surveillance (IBG-RCS) est une méthode utilisée en écologie appliquée afin de déterminer la qualité 
biologique d'un cours d'eau se basant sur l'étude de sa communauté macrobenthique.

Le présent travail a donc pour objectif de déterminer, à la lumière des résultats obtenus concernant essentiellement les prélèvements de la macrofaune benthique et le calcul de l'indice biologique global des réseaux de contrôle et de surveillance, l'état général du bassin versant d’Oued Beht.

\section{Matériel et méthode}

\section{Milieu d'étude}

Le bassin versant d'Oued Beht se situe au Nord-Ouest du Maroc et occupe une superficie d'environ $4500 \mathrm{Km}^{2}$ au niveau de la partie Sud-Ouest du bassin du Sébou. Il est limité au Nord par la plaine du Gharb et le plateau de Meknès, au Sud par le bassin d'Oum-Erbia, à l'Ouest par le bassin de Bouregreg et à l'Est par le Moyen Atlas. Ses limites s'inscrivent entre les méridiens $5^{\circ}$ et $6^{\circ}$ Ouest et les parallèles $33^{\circ}$ et $34^{\circ}$ Nord (Abdallaoui, 1998) (figure 1).

Ce cours d'eau prend naissance dans la zone d'Azrou où plusieurs oueds de cette région confluent, notamment les oueds Tigrigra et Ifrane. Tout en traversant le plateau central, l'oued Beht s'alimente par les apports de nombreux oueds tels que: Bou Achouch, Kharouba, Ouchket, El Kell, Berrajline, D’kor et Chébi (Haut-Commissariat aux Eaux et Forêts et à la Lutte Contre la Désertification [HCEFLCD], 2006; Sahili and Makhokhi, 1987). Avant de rejoindre le Sebou, l'Oued Beht reçoit l'Oued R'Dom (Agence du Bassin Hydraulique du Sebou [ABHS], 2010).

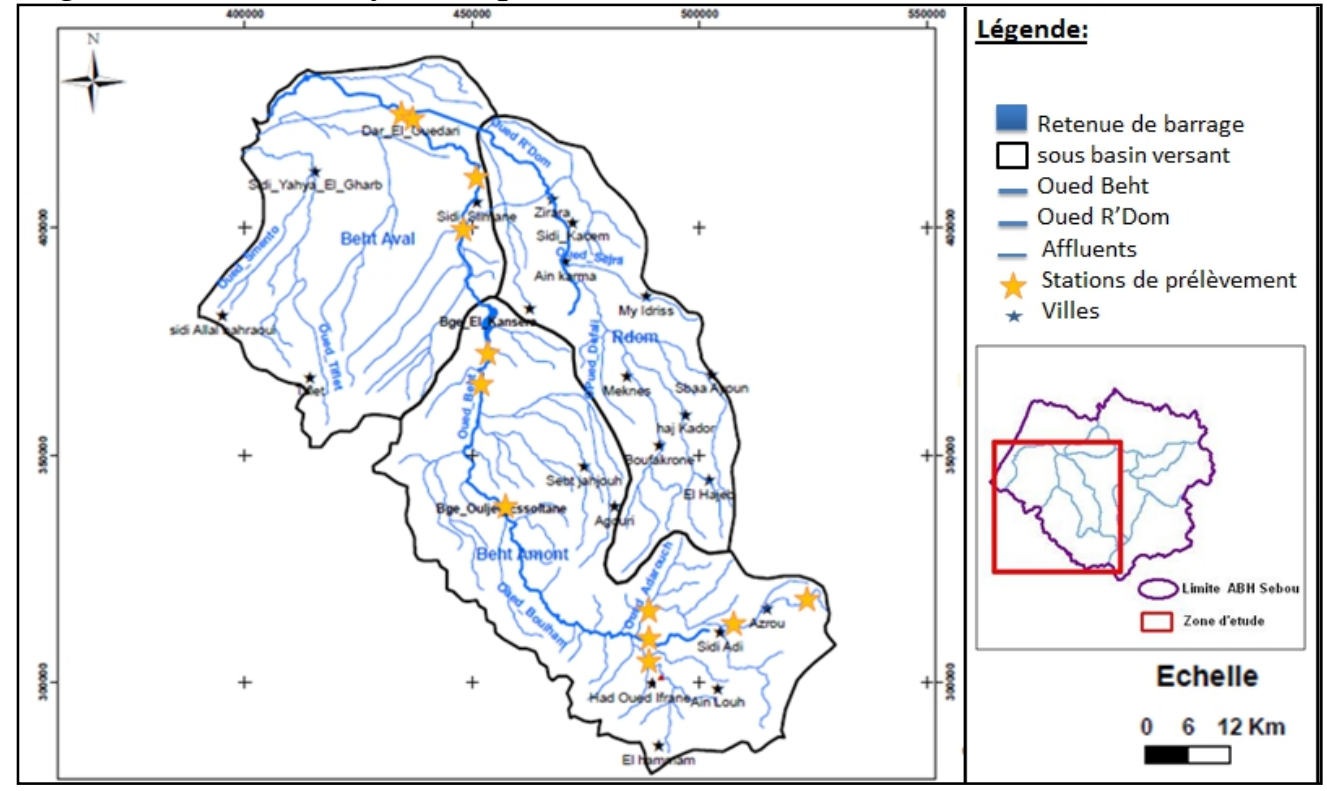

Figure 1. Présentation de la zone d'étude

Figure 1. The studied zone 
Sur le plan géologique, le bassin versant d'oued Beht est installé sur plusieurs domaines géologiques et structuraux. On distingue selon HCEFLCD (2006) et Mint Chevie (2011) :

- Haut beht : la méséta centrale à formations primaires et le causse moyen atlasique qui reporte les caractéristiques géologiques du Moyen Atlas (apparition des calcaires jurassiques plus des épandages basaltiques d'âge quaternaire).

- Moyen Beht : le plateau de Méknès et les rides prérifaines, composée par un complexe marneux tertiaire. Lequel complexe surmonte une série marno-calcaire et dolomique du jurassique.

- Bas Beht: la plaine du Gharb et le secteur de la Maamoura Méridionale. Les affleurements du complexe de Maamoura sont représentés par des sables gréseux du Plio-quaternaire couvrant des marnes miocènes.

\section{Stations d'échantillonnage}

Notre campagne de prélèvement de la macrofaune benthique sur le bassin versant d'oued Beht a été réalisée pendant la période Mai-Juin de l'année 2014 au niveau de douze stations dont le positionnement est présenté sur la figure 1. Les stations 1 (Ras El Ma), 2 (Sidi Elmokhfi), 3 (Adarouch), 4 (Amghas), 5 (Had oued Ifrane) se situent sur le Beht amont. Les stations 6 (Ouljet Sultane), 7 (amont rejet Khémisset), 8 (aval rejet Khémisset) et 9 (Dar Bel Amri) représentent le Beht moyen. Les dernières trois stations (Sidi Slimane, amont et aval Dar El Gueddari) se situent sur le Beht aval.

Tableau 1. Coordonnées des stations de prélèvements

Table 1. Stations coordinates

\begin{tabular}{|c|c|c|}
\hline \multirow{2}{*}{ Station } & \multicolumn{2}{|c|}{ Les coordonnées } \\
\cline { 2 - 3 } & 5 & Y \\
\hline Ras El Ma & 523314 & 318561 \\
\hline Sidi Elmokhfi & 507676 & 311800 \\
\hline Adarouch & 487796 & 315183 \\
\hline Amghas & 495540 & 308987 \\
\hline aval Had oued Ifrane & 491369 & 299775 \\
\hline Ouljet sultane & 452250 & 338050 \\
\hline amont Khémisset & 450816 & 364815 \\
\hline aval Khémisset & 450300 & 369150 \\
\hline Dar Bel Amri & 447720 & 439970 \\
\hline Sidi slimane & 449350 & 415180 \\
\hline amont Dar El Gueddari & 436175 & 424152 \\
\hline aval Dar El Gueddari & 435592 & 424510 \\
\hline
\end{tabular}

\section{Echantillonnage biologique}

Afin d'évaluer la qualité biologique de notre zone d'étude nous nous sommes basé sur l'indice biologique global des réseaux de contrôle et de surveillance comme un instrument de surveillance intégrée de l'état écologique d'oued Beht. Les prélèvements de la macrofaune benthique sont 
fait conformément au protocole relatif à cet indice, publié par l'AFNOR en 2009 (Agence Française de Normalisation [AFNOR], 2009) sous la référence « norme XP T90-333 ».

Ce protocole introduit récemment au Maroc (2011), consiste dans sa première étape, en la réalisation de 12 prélèvements de la macrofaune benthique sur les différents substrats dominants et marginaux présents dans le milieu aquatique moyennant les filets Surber $\left(1 / 20 \mathrm{~m}^{2}\right)$ et Troubleau selon l'accessibilité des différents substrats. Les prélèvements, fixés par l'alcool à $70^{\circ}$, sont ensuite transportés au laboratoire pour faire l'objet des opérations de tri et de détermination de la deuxième étape du protocole. La détermination des différents taxons se fait sous une loupe binoculaire et à l'aide de clés de détermination.

Le calcul de la note dans le protocole de l'indice biologique global des réseaux de controle et de surveillance est basé sur les mêmes fondements que pour le calcul de la note de l'Indice Biologique Global Normalisé (IBGN) (Agences françaises de l'eau, 2000). Cette dernière est calculée à partir d'un tableau d'analyse combinant la variété taxonomique (abscisse) et le groupe indicateur le plus “'polluosensible’' (ordonnée). La détermination du groupe indicateur (GI) s’effectue en prospectant l’ordonnée du tableau de haut en bas (GI 9 au GI 1) et en arrêtant l'examen à la première présence significative ( $\mathrm{n} \geq 3$ individus ou $\mathrm{n} \geq 10$ individus) d'un taxon du répertoire en ordonnée du tableau 2 (Agences françaises de l'eau, 2000).

La note de qualité hydrobiologique variant de 0 à 20. La gamme de la qualité proposée (cas général en France) est consignée dans le tableau 3.

Tableau 2. Calcul de l'indice biologique global normalisé (IBGN) (Archaimbault and Dumont, 2010)

Table 2. IBGN calculation

\begin{tabular}{|c|c|c|c|c|c|c|c|c|c|c|c|c|c|c|c|}
\hline Classe de variété & & 14 & 13 & 12 & 11 & 10 & 9 & 8 & 7 & 6 & 5 & 4 & 3 & 2 & 1 \\
\hline Nbr de familles & & $\geq 5$ & $40-$ & 44- & $40-$ & 36- & $32-$ & $28-$ & $24-$ & $20-$ & $16-$ & $12-$ & 9- & 6- & $\leq$ \\
\hline Taxons indicateurs (pollvo-sensibilité & & & 45 & 41 & 37 & & 29 & & 21 & 17 & 13 & 10 & 7 & 4 & 3 \\
\hline $\begin{array}{l}\text { Chloroperlidae -Perlidae } \\
\text { Perlodidae - Taeniopterygidae }\end{array}$ & 9 & 20 & 20 & 20 & 19 & 18 & 17 & 16 & 15 & 14 & 13 & 12 & 11 & 10 & 9 \\
\hline $\begin{array}{l}\text { Capniidae- Brachycentridae } \\
\text { Odontoceridae- Philopotamidae }\end{array}$ & 8 & 20 & 20 & 19 & 18 & 17 & 16 & 15 & 14 & 13 & 12 & 11 & 10 & 9 & 8 \\
\hline $\begin{array}{l}\text { Leuctridae - Glossosomatidae } \\
\text { Beraeidae - Goeridae -Leptophelbidae }\end{array}$ & 7 & 20 & 19 & 18 & 17 & 16 & 15 & 14 & 13 & 12 & 11 & 10 & 9 & 8 & 7 \\
\hline $\begin{array}{l}\text { Nemouridae - Lepidostomatidae } \\
\text { Sericostomatidae - Ephemeridae }\end{array}$ & 6 & 19 & 18 & 17 & 16 & 15 & 14 & 13 & 12 & 11 & 10 & 9 & 8 & 7 & 6 \\
\hline $\begin{array}{l}\text { Hydroptilidae - Heptageniidae } \\
\text { Polymitarcidae - Potamanthidae }\end{array}$ & 5 & 18 & 17 & 16 & 15 & 14 & 13 & 12 & 11 & 10 & 9 & 8 & 7 & 6 & 5 \\
\hline $\begin{array}{l}\text { Leptoceridae - Polycentropodidae } \\
\text { Psychomyidae - Rhyacophilidae }\end{array}$ & 4 & 17 & 16 & 15 & 14 & 13 & 12 & 11 & 10 & 9 & 8 & 7 & 6 & 5 & 4 \\
\hline $\begin{array}{l}\text { Limnephilidae- Ephemerellidae } \\
\text { Hydropsychidae - Aphelocheiridae }\end{array}$ & 3 & 16 & 15 & 14 & 13 & 12 & 11 & 10 & 9 & 8 & 7 & 6 & 5 & 4 & 3 \\
\hline $\begin{array}{l}\text { Baetidae - Caenidae } \\
\text { Elmidae - Gammaridae - Mollusques }\end{array}$ & 2 & 15 & 14 & 13 & 12 & 11 & 10 & 9 & 8 & 7 & 6 & 5 & 4 & 3 & 2 \\
\hline $\begin{array}{l}\text { Chironomidae - Asellidae } \\
\text { Achètes - Oligochètes }\end{array}$ & 1 & 14 & 13 & 12 & 11 & 10 & 9 & 8 & 7 & 6 & 5 & 4 & 3 & 3 & 1 \\
\hline
\end{tabular}


Tableau 3. Gamme de qualité IBGN (Archaimbault and Dumont, 2010)

Table 3. Range of quality IBGN

\begin{tabular}{|c|c|c|c|c|c|}
\hline IBGN & $>=17$ & $16-13$ & $12-9$ & $8-5$ & $<=4$ \\
\hline Qualité & très bonne & bonne & moyen & médiocre & mauvaise \\
\hline Couleur & bleu & vert & jaune & orange & rouge \\
\hline
\end{tabular}

\section{Résultats et discussion}

\section{Inventaire faunistique et répartition des principaux groupes faunistiques Inventaire faunistique du Beht}

Le tableau 4 dresse les différents taxons trouvés dans les stations d'échantillonnage de notre zone d'étude.

Tableau 4. Inventaire de la macrofaune benthique récoltée dans l’Oued Beht

Table 4. Invertebrates inventory of Beht's river

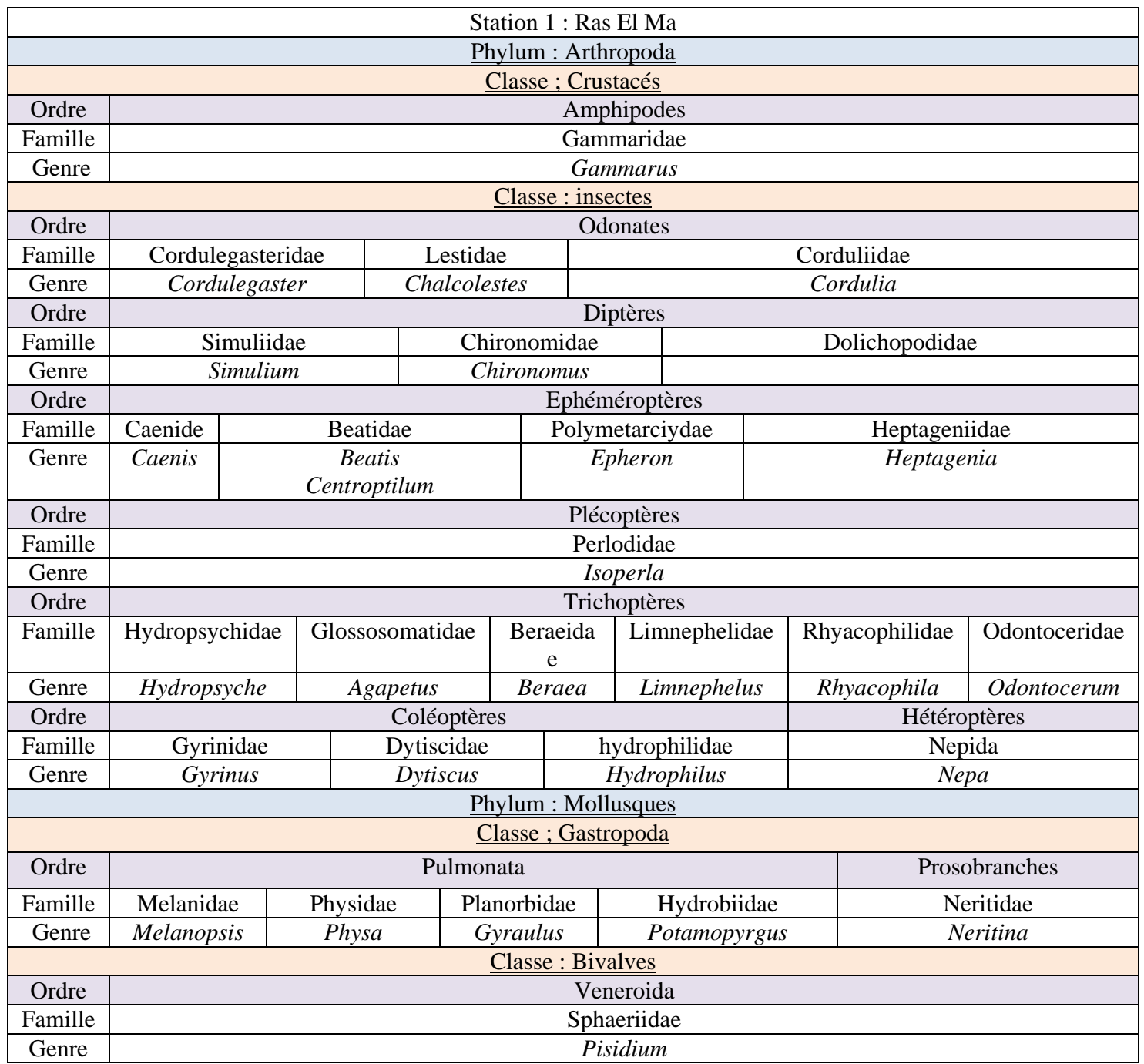




\begin{tabular}{|c|c|c|}
\hline \multicolumn{2}{|c|}{ Phylum : Annélides } \\
\hline \multicolumn{2}{|c|}{ Classe ; Oligochètes } & Classe : Achètes \\
\hline Ordre & Haplotaxida & Rhynchobdellida \\
\hline Famille & Haplotaxidae & Glossiphonidae \\
\hline Genre & Haplotaxis & Helobdella \\
\hline
\end{tabular}

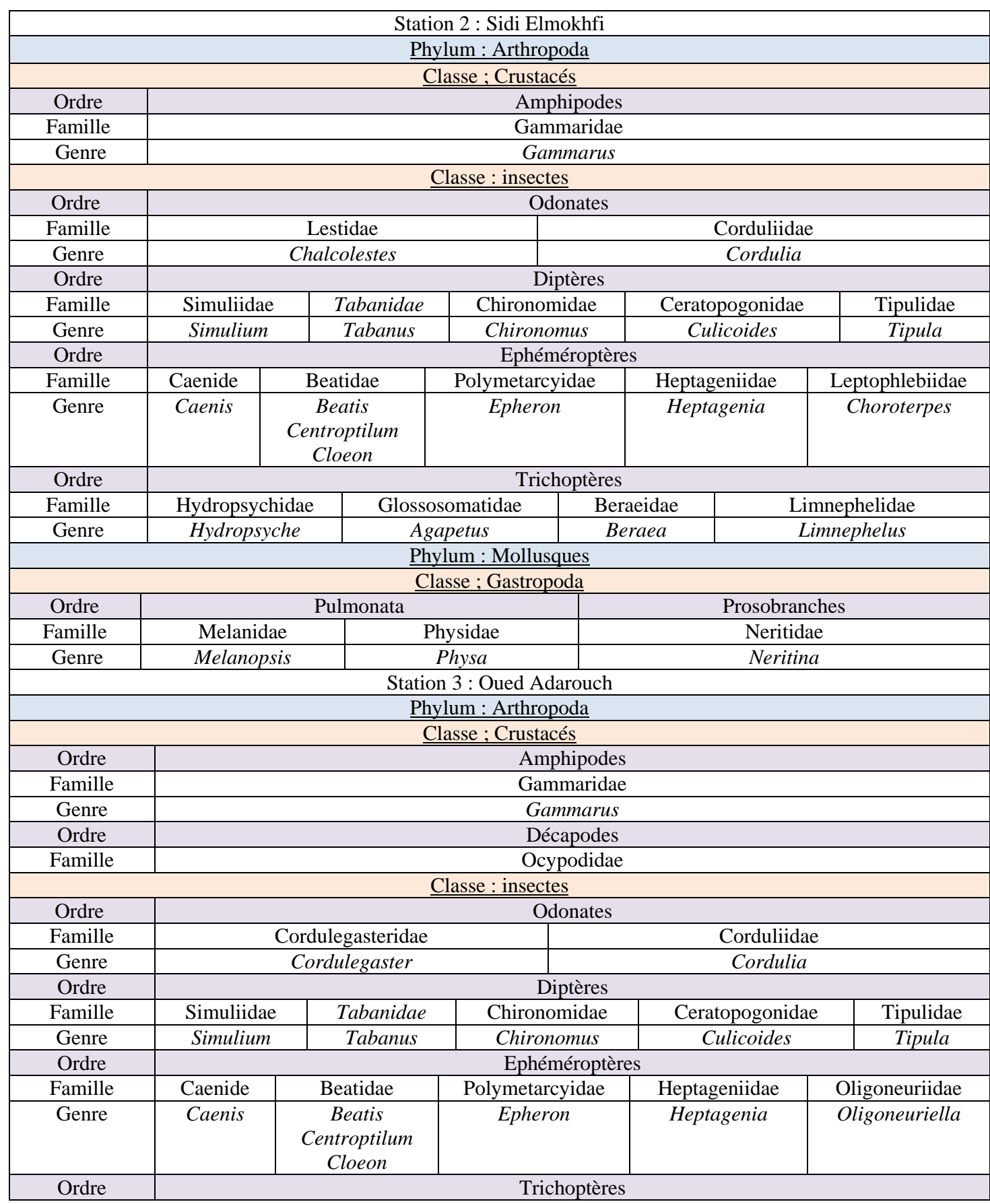




\begin{tabular}{|c|c|c|c|}
\hline Famille & Hydropsychidae & Limnephilidae \\
\hline Genre & \multicolumn{3}{|c|}{ Hydropsyche } \\
\hline \multicolumn{3}{|c|}{ Phylum : Mollusques } \\
\hline \multicolumn{3}{|c|}{ Classe; Gastropoda } \\
\hline Ordre & Pulmonata & Prosobranches \\
\hline Famille & Melanidae & Physidae & Neritidae \\
\hline Genre & Melanopsis & Physa & Neritina \\
\hline
\end{tabular}

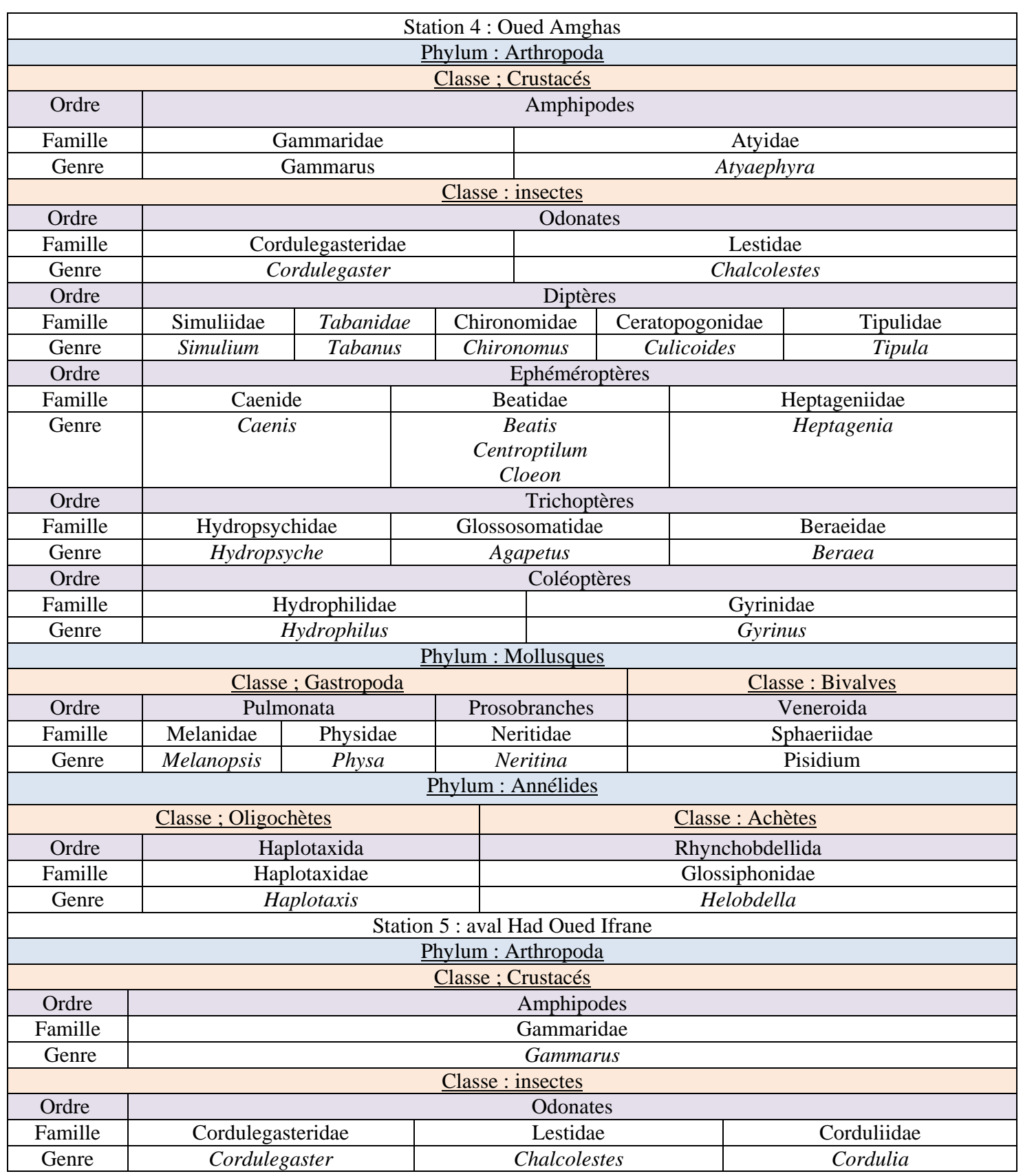




\begin{tabular}{|c|c|c|c|c|c|c|}
\hline Ordre & \multicolumn{6}{|c|}{ Diptères } \\
\hline Famille & Simuliidae & Tabanidae & \multicolumn{2}{|c|}{ Chironomidae } & Ceratopogonidae & Tipulidae \\
\hline Genre & Simulium & Tabanus & & ironomus & Culicoides & Tupila \\
\hline Ordre & \multicolumn{4}{|c|}{ Ephéméroptères } & \multicolumn{2}{|c|}{ Trichoptères } \\
\hline Famille & \multicolumn{2}{|c|}{ Beatidae } & \multicolumn{2}{|c|}{ Heptageniidae } & \multicolumn{2}{|c|}{ Hydropsychidae } \\
\hline Genre & \multicolumn{2}{|c|}{$\begin{array}{c}\text { Centroptilum } \\
\text { Cloeon }\end{array}$} & \multicolumn{2}{|c|}{ Heptagenia } & \multicolumn{2}{|c|}{ Hydropsyche } \\
\hline \multicolumn{7}{|c|}{ Phylum : Mollusques } \\
\hline \multicolumn{7}{|c|}{ Classe ; Gastropoda } \\
\hline Ordre & \multicolumn{6}{|c|}{ Pulmonata } \\
\hline Famille & \multicolumn{3}{|c|}{ Melanidae } & \multicolumn{3}{|c|}{ Physidae } \\
\hline Genre & \multicolumn{2}{|c|}{ Melanopsis } & & \multicolumn{3}{|c|}{ Physa } \\
\hline \multicolumn{7}{|c|}{ Phylum : Annélides } \\
\hline \multicolumn{7}{|c|}{ Classe; Oligochètes } \\
\hline Ordre & \multicolumn{6}{|c|}{ Haplotaxida } \\
\hline Famille & & & & Iaplotaxidae & & \\
\hline Genre & & & & Haplotaxis & & \\
\hline & & Stat & & et Sultane & & \\
\hline & & & & hropoda & & \\
\hline & & & & sectes & & \\
\hline Ordre & & & & Odonates & & \\
\hline Famille & Cor & asteridae & & & Corduliidae & \\
\hline Genre & & egaster & & & Cordulia & \\
\hline Ordre & & & & Diptères & & \\
\hline Famille & Tabanidae & Chirono & & Ceratopogonidae & Ptyc & ridae \\
\hline Genre & Tabanus & Chiron & & Culicoides & Pty & era \\
\hline Ordre & & & & léméroptères & & \\
\hline Famille & Caenide & Beatidae & & Polymetarcyidae & Hepta & \\
\hline Genre & Caenis & $\begin{array}{r}\text { Beatis } \\
\text { Centroptil } \\
\text { Cloeon }\end{array}$ & & Epheron & Hept & \\
\hline Ordre & & tères & & & Hétéroptères & \\
\hline Famille & $\mathrm{Hyc}$ & chidae & & & Nepidae & \\
\hline Genre & & syche & & & Nepa & \\
\hline & & Station & 101 & jejet Khémisset & & \\
\hline & & & 1: & hropoda & & \\
\hline & & & e; & istacés & & \\
\hline Ordre & & & & mphipodes & & \\
\hline Famille & & & & ammaridae & & \\
\hline Genre & & & & Iammarus & & \\
\hline & & & $\mathrm{se}$ & sectes & & \\
\hline Ordre & & & & Odonates & & \\
\hline Famille & & dae & & & Corduliidae & \\
\hline Genre & & lestes & & & Cordulia & \\
\hline Ordre & & & & Diptères & & \\
\hline Famille & & lidae & & & Chironomidae & \\
\hline Genre & & lium & & & Chironomus & \\
\hline Ordre & & & & iéméroptères & & \\
\hline Famille & Caenide & Beatic & & Polymetarcyidae & Hept & idae \\
\hline Genre & Caenis & $\begin{array}{r}\text { Beat } \\
\text { Centrop } \\
\text { Cloec }\end{array}$ & & Epheron & $\mathrm{Hep}$ & \\
\hline
\end{tabular}




\begin{tabular}{|c|c|c|c|c|}
\hline Ordre & \multicolumn{4}{|c|}{ Trichoptères } \\
\hline Famille & \multicolumn{2}{|c|}{ Hydropsychidae } & \multicolumn{2}{|r|}{ Limnephilidae } \\
\hline Genre & \multicolumn{2}{|c|}{ Hydropsyche } & \multicolumn{2}{|r|}{ Limnephilus } \\
\hline \multicolumn{5}{|c|}{ Phylum : Mollusques } \\
\hline \multicolumn{5}{|c|}{ Classe ; Gastropoda } \\
\hline Ordre & \multicolumn{2}{|c|}{ Pulmonata } & \multicolumn{2}{|r|}{ Prosobranches } \\
\hline Famille & Melanidae & Physidae & \multicolumn{2}{|r|}{ Neritidae } \\
\hline Genre & Melanopsis & Physa & \multicolumn{2}{|r|}{ Neritina } \\
\hline \multicolumn{5}{|c|}{ Classe : Bivalves } \\
\hline Ordre & \multicolumn{2}{|c|}{ Veneroida } & \multicolumn{2}{|r|}{ Unionoida } \\
\hline Famille & \multirow{2}{*}{\multicolumn{2}{|c|}{$\begin{array}{l}\text { Sphaeriidae } \\
\text { Pisidium }\end{array}$}} & \multicolumn{2}{|r|}{ Unionidae } \\
\hline Genre & & & \multicolumn{2}{|r|}{ Unio } \\
\hline \multirow{2}{*}{\multicolumn{5}{|c|}{$\begin{array}{l}\text { Phylum : Annélides } \\
\text { Classe : Oligochètes }\end{array}$}} \\
\hline & & & iètes & \\
\hline Ordre & & 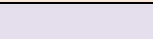 & lotaxida & \\
\hline Famille & & & otaxidae & \\
\hline Genre & & & blotaxis & \\
\hline & & Station $8: \mathrm{a}$ & Khémisset & \\
\hline & & Phylun & poda & \\
\hline & & Class & cés & \\
\hline Ordre & & & hipodes & \\
\hline Famille & & & maridae & \\
\hline Genre & & & Imarus & \\
\hline & & Clas & & \\
\hline Ordre & & & onates & \\
\hline Famille & & & & Corduliidae \\
\hline Genre & & & & Cordulia \\
\hline Ordre & & & tères & \\
\hline Famille & Simuliidae & Tipulidae & Chironomidae & Tabanidae \\
\hline Genre & Simulum & Tipula & Chironomus & Tabanus \\
\hline Ordre & & & éroptères & \\
\hline Famille & Caenide & & & Polymetarcyidae \\
\hline Genre & Caenis & & & Epheron \\
\hline Ordre & & & optères & \\
\hline Famille & Hyd & & & Limnephelidae \\
\hline Genre & & & & Limnephilus \\
\hline & & Phylun & ques & \\
\hline & & Classe & oda & \\
\hline Ordre & & hata & & Prosobranches \\
\hline Famille & Melanidae & Physi & & Neritidae \\
\hline Genre & Melanopsis & Phys & & Neritina \\
\hline & & Phylui & ides & \\
\hline & & Classe & ètes & \\
\hline Ordre & & & lotaxida & \\
\hline Famille & & & otaxidae & \\
\hline Genre & & & plotaxis & \\
\hline & & Station 9 & I Amri & \\
\hline & & Phylun & poda & \\
\hline & & Class & cés & \\
\hline Ordre & & & hipodes & \\
\hline Famille & & & & Atyidae \\
\hline
\end{tabular}




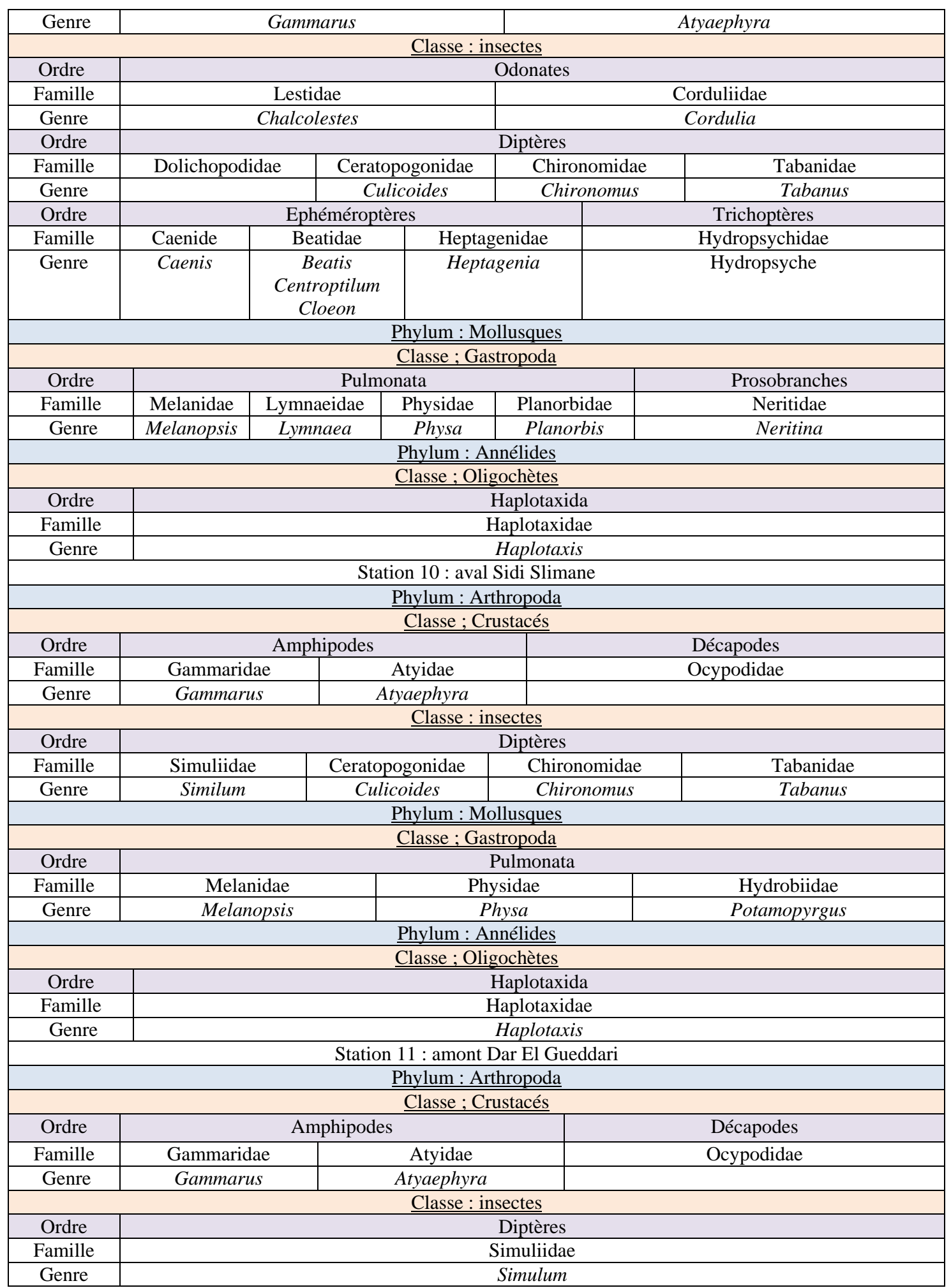




\begin{tabular}{|c|c|c|c|}
\hline \multicolumn{4}{|c|}{ Phylum : Mollusques } \\
\hline Ordre & Classe ; Gastropoda & Classe : Bivalves \\
\hline Famille & Pulmonata & Veneroida \\
\hline Genre & Viviparidae & Sphaeridae \\
\hline \multicolumn{4}{|c|}{ Phylum : Annélides } \\
\hline Viviparus & Classe ; Oligochètes \\
\hline Ordre & \multicolumn{3}{c|}{ Haplotaxida } \\
\hline Famille & Haplotaxidae \\
\hline Genre & \multicolumn{3}{c|}{ Haplotaxis } \\
\hline
\end{tabular}

\begin{tabular}{|c|c|c|c|}
\hline \multicolumn{4}{|c|}{ Station 12 : aval Dar El Gueddari } \\
\hline \multicolumn{4}{|c|}{ Phylum : Arthropoda } \\
\hline \multicolumn{4}{|c|}{ Classe; Crustacés } \\
\hline Ordre & \multicolumn{2}{|c|}{ Amphipodes } & Décapodes \\
\hline Famille & Gammaridae & Atyidae & Ocypodidae \\
\hline Genre & Gammarus & Atyaephyra & \\
\hline \multicolumn{4}{|c|}{ Phylum : Mollusques } \\
\hline \multicolumn{3}{|c|}{ Classe ; Gastropoda } & Classe : Bivalves \\
\hline Ordre & \multicolumn{2}{|c|}{ Pulmonata } & Veneroida \\
\hline Famille & Viviparidae & Melanidae & Sphaeridae \\
\hline Genre & Viviparus & Melanopsis & Pisidium \\
\hline
\end{tabular}

\section{Répartition des principaux groupes faunistiques}

Les communautés macrozoobenthiques sont très sensibles à la variabilité environnementale (Arienzo et al., 2001). De ce fait, la diversité de la macrofaune benthique et son abondance dans un système lotique donné peuvent apporter des informations importantes sur la qualité de ce milieu.

La répartition spatiale des différents groupes des macroinvertébrés benthiques inventoriés montre une dominance des groupes faunistiques présentant des niveaux moyens à très tolérants vis-à-vis la pollution organique. C'est le cas notamment des groupes Annélides, Crustacés et Mollusques, Diptères et Odonates présents abondamment dans la majorité des stations d'échantillonnage (figure 2).

Le spectre faunistique connait également la présence d’autres groupes faunistiques nommément les Plécoptères, Trichoptères et Ephéméroptères. Lesquels groupes sont considérés, en général, comme des bons indicateurs des eaux propres (Kolkwitz and Marsson, 1909). Cependant certaines espèces (appartenant notamment aux familles Hydropsychidae et Limnephilidae pour le groupe Trichoptères et les familles Baetidae et Caenidae du groupe Ephéméroptères) peuvent tolérer des niveaux de contamination organiques élevés (Hellawell, 1986).

L'étude des différents spectres faunistiques relatifs aux stations d'échantillonnage relève également une diminution de la diversité de la macrofaune benthique des stations situées dans le Beht amont vers les stations situées dans la zone avale du bassin versant étudié. Cette diminution 
est exprimée par la disparition de certains taxons dont les plus importants sont les suivants :

- $\quad$ les Plécoptères qui ne sont présents qu’au niveau d'une seule station qui est la station $\mathrm{N}^{\circ} 1$ (Ras Elma) située dans une zone montagnarde dans la région d'Ifrane (Moyen Atlas) ;

- les Trichoptères et Ephéméroptères au niveau des 3 dernières stations situées dans la zone avale du bassin versant d’Oued Beht.

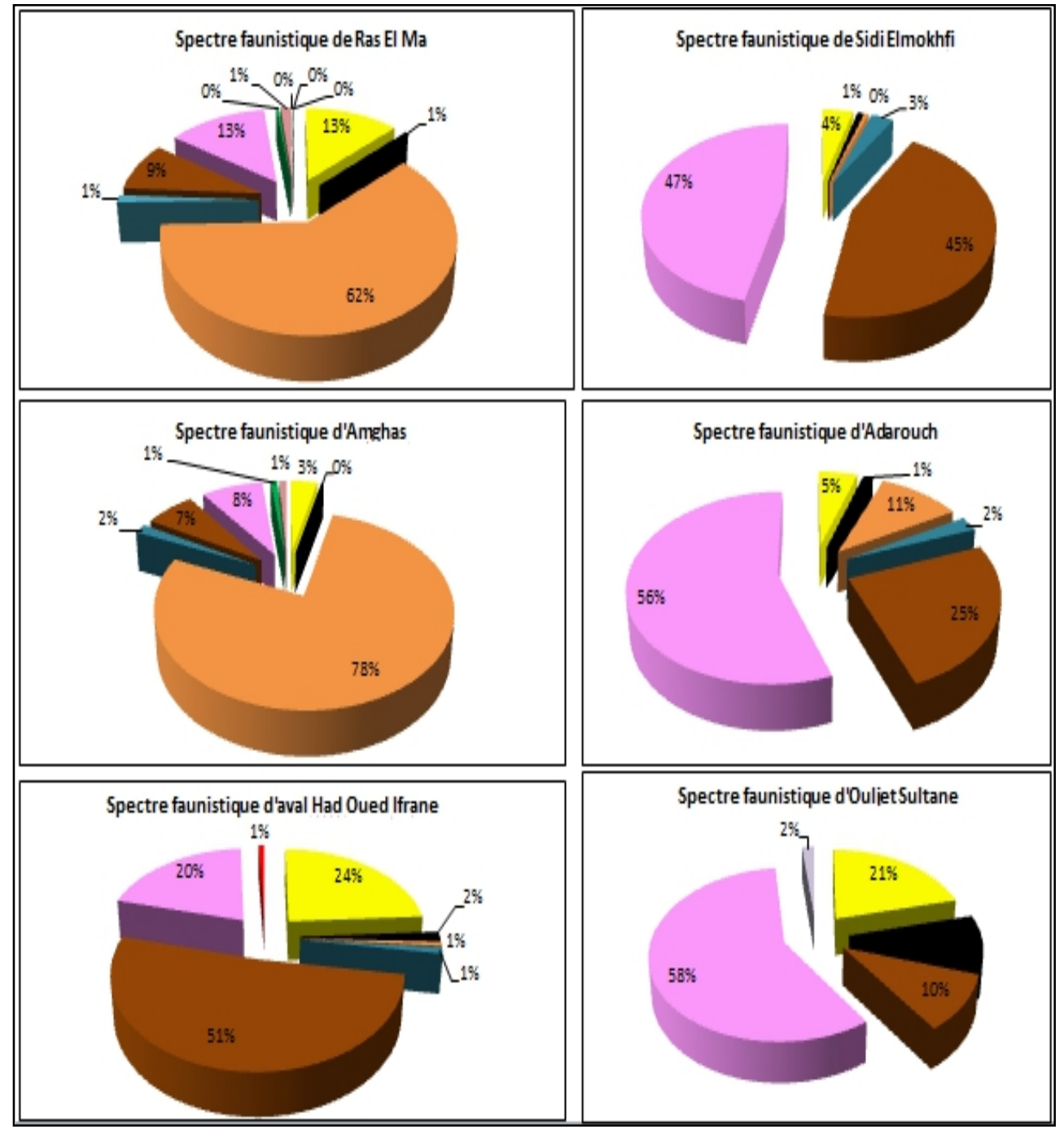

Figure 2 (a). Spectre faunistique de l'Oued Beht des stations 1 à 6

Figure 2 (a). Zonal spectrum of stations from 1 to 6 of Beht's river 

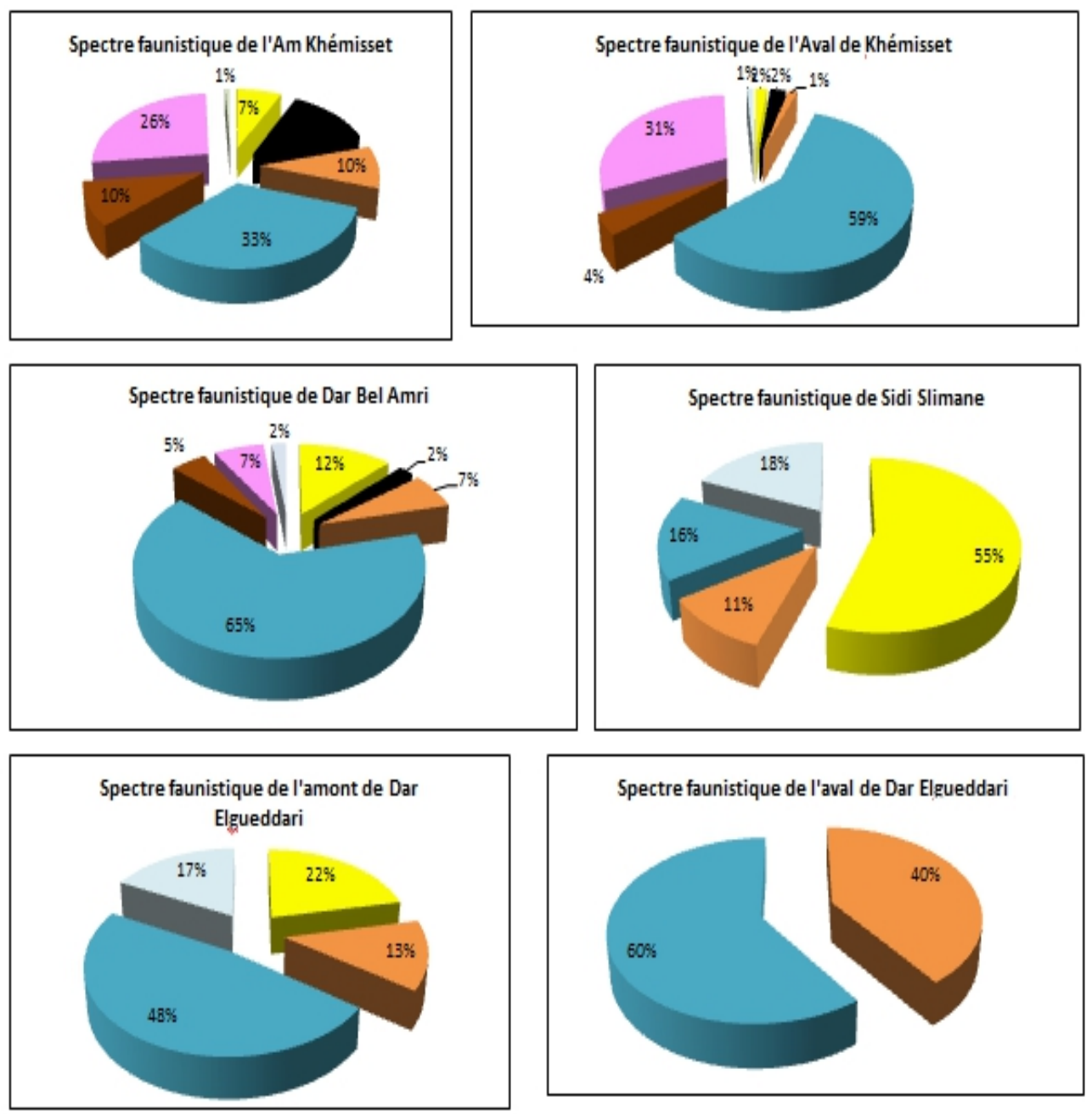

Figure 2 (b). Spectre faunistique de l'Oued Beht des stations de 7 à 12 Figure 2 (b). Zonal spectrum of stations from 7 to 12 of Beht's river

Avec:

\begin{tabular}{|l|lll|}
\hline Diptères & Odonates & Mollusques & Trichoptères \\
Ephéméroptères & Coléoptères & Achètes & Oligochètes \\
Hétéroptères & Plécoptères & Crustacés & \\
\hline
\end{tabular}

\section{Calcul de la note d'indice IBG-RCS}

5 stations ont été prospectées au niveau du Beht amont. Il s'agit notamment des stations Ras El Ma, Amghas, Adarouch, Sidi Elmokhfi et aval Had Oued Ifrane. Les stations suivantes Adarouch, Sidi Elmokhfi et aval Had Oued Ifrane sont caractérisée par une valeur indicielle oscillant entre 9 et 12 témoignant d'une eau de moyenne qualité. En effet, la région d'Adarouch ou se situe la première station est d'une vocation purement agricole (environ 1500 hectares de terres agricoles, ranchs d'élevage du 
bétail et abattoirs) mais qui a également connue l'installation d'une décharge publique non contrôlée en 2014. Ces différentes activités conduisent à la diffusion de divers polluants organiques dont l'accumulation est toxique visà-vis l'environnement en général et qui sont probablement à l'origine de la dégradation du système lotique (oued Adarouch). Les deux autres stations sont situées en aval des centres urbains (ville d'Azrou et village Had oued Ifrane) et en conséquent en aval des déversements de polluants organiques d'origine domestique conduisant à la détérioration de la qualité biologique des oueds dans cette région.

La station Amghas est située dans une zone considérée comme loin d'une fréquentation humaine perturbante, elle est créditée alors des eaux de bonne qualité avec une note indicielle égale à 13. La station Ras El Ma située dans la zone d'Ifrane est créditée de la meilleure note indicielle dans le bassin versant du Beht (17/20) et présente ainsi une eau d'excellente qualité.

Le Beht moyen est représenté par 4 station ; Ouljet Sultane, amont et aval rejet Khémisset et Dar Bel Amri. Les 3 dernières stations indiquent des eaux de moyenne qualité avec une note indicielle de 9/20 au niveau de la station aval rejet khémisset et de 10/20 au niveau des 2 autres stations. Tout comme les autres stations se situant en aval des centres urbains, ces stations reçoivent des rejets domestiques ayant un rôle prépondérant dans la dégradation de la qualité biologique du cours d'eau.

La station Ouljet Sultane est considérée comme située loin des grandes perturbations humaines, cependant elle présente une note indicielle de 8/20 et ainsi des eaux de qualité médiocre. Ce résultat peut d'être causé par l'extraction des matériaux alluvionnaires (substrats pour l'installation de la macrofaune benthique) à finalité de leur utilisation dans la construction du barrage Ouljet Sultane. En effet, plusieurs auteurs ont démontré les effets négatifs des extractions de matériaux alluvionnaires sur l'environnement aquatique en général et sur les peuplements benthiques en particulier, on cite par exemple les travaux des agences de l'eau en France (2000).

Finalement, les stations Sidi Slimane, amont et aval Dar El Gueddari se situant sur le Beht aval (en aval du barrage Elkensera) indiquent des eaux de médiocre à mauvaise qualité biologique et ce avec des notes indicielles entre $3 / 20$ et $5 / 20$. Ces stations reçoivent divers polluants organiques domestiques provenant des centres urbains (Sidi Slimane et Dar El Gueddari) et agricoles (agricultures vivrières sur les bords des oueds et déversements des abattoirs). On note que les eaux des présentes stations sont très riches en algues (eutrophisation du système lotique) avec une odeur très désagréable causée par la dégradation de la matière organique par les bactéries, une présence de plusieurs cadavres de vaches dans le lit d'oued et la présence de nombreuses machines de pompage des eaux de l'oued Beht pour l'irrigation des petites parcelles cultivées au niveau des bords. 
Par ailleurs la diminution de la diversité de la macrofaune benthique cause de la diminution de la valeur de l'indice biologique dans ces dernières stations peut revenir également aux changements hydrologiques et climatiques qui caractérisent cette zone du bassin versant d'Oued Beht. En effet, le Beht aval s'installe sur la plaine du Gharb en aval d'un ouvrage hydro-électrique (barrage Elkensera), paramètres qui peuvent être considérés comme stress conduisant à un changement quantitatif mais également qualitatif vis-à-vis la macrofaune benthique présente dans ce milieu.

Tableau 4. Calcul de la note de l'Indice Biologique Global des Réseaux de Contrôle et de Surveillance

Table 4. Index calculation

\begin{tabular}{|c|c|c|c|c|}
\hline Nom de la station & $\begin{array}{c}\text { Richesse taxonomique } \\
\text { (Nombre de familles) }\end{array}$ & $\begin{array}{c}\text { Groupe } \\
\text { indicateur }\end{array}$ & $\begin{array}{c}\text { Note } \\
\text { indicielle }\end{array}$ & $\begin{array}{c}\text { Qualité de la } \\
\text { station }\end{array}$ \\
\hline Ras El Ma & 30 & 9 & $17 / 20$ & Très bonne \\
\hline Amghas & 23 & 7 & $13 / 20$ & Bonne \\
\hline Adarouch & 19 & 5 & $10 / 20$ & Moyenne \\
\hline Sidi al mokhfi & 20 & 7 & $12 / 20$ & Moyenne \\
\hline aval had oued Ifrane & 16 & 5 & $9 / 20$ & Moyenne \\
\hline Ouljet Sultane & 12 & 5 & $8 / 20$ & Médiocre \\
\hline amont rejet Khémisset & 17 & 5 & $10 / 20$ & Moyenne \\
\hline aval rejet Khémisset & 16 & 5 & $9 / 20$ & Moyenne \\
\hline Dar bel Amri & 18 & 5 & $10 / 20$ & Moyenne \\
\hline Sidi Slimane & 11 & 2 & $5 / 20$ & Médiocre \\
\hline amont Dar El Gueddari & 7 & 2 & $4 / 20$ & Mauvaise \\
\hline aval Dar El Gueddari & 6 & 2 & $3 / 20$ & Mauvaise \\
\hline
\end{tabular}

\section{Conclusion}

Cette étude est une contribution à l'évaluation de la qualité biologique du réseau hydrologique du bassin versant d'Oued Beht (sous bassin très important du bassin hydrographique du Sebou) suivant la démarche biocénotique prenant comme moyen l'Indice Biologique Global des Réseaux de Contrôle et de Surveillance (IBG-RCS). Les résultats de l'analyse des prélèvements biologiques effectués sur 12 stations choisies sur l'Oued Beht durant la période Mai-Juin 2014 montrent la diminution de la valeur de l'indice biologique de l'amont vers l'aval du cours d'eau par diminution de la richesse en macrofaune benthique. La macrofaune benthique de ce cours d'eau est également dominé par les groupes faunistiques polluo-résistants. Ces résultats peuvent être expliqués par la combinaison de plusieurs facteurs stressants naturels ou d'origine anthropiques dans la zone avale du bassin étudié notamment le changement du climat (allant de l'humide vers le semi-aride) et l'action anthropique reflétée par l'installation des ouvrages hydro-électriques et la pollution due spécifiquement à l'agriculture et aux rejets domestiques ou encore la présence de décharges publiques non contrôlées et/ ou sauvages. 


\section{References:}

1. Abdallaoui, A. (1998). Contribution à l'étude du phosphore et des métaux lourds contenus dans les sédiments et de leur influence sur les phénomènes d'eutrophisation et de la pollution: Cas du bassin versant de l'Oued Beht et de la retenue de barrage El Kansera.

2. Agence du Basin Hydraulique du Sebou [ABHS]. (2010). Etude de mise en place d'un système d'alerte aux crues et de télémesure dans le bassin du Beht.

3. Agence Française de Normalisation [AFNOR]. (2009). Détermination de l'indice biologique global des réseaux de controle et de surveillance.

4. Agences françaises de l'eau (2000). Indice biologique global normalisé, guide technique.

5. Archaimbault, V., and Dumont, B. (2010). L'indice biologique global normalisé (IBGN): principes et évolution dans le cadre de la directive cadre Européenne sur l'eau. 36-39.

6. Arienzo, M., Adamo, P., Bianco, R., and Violante, P. (2001). Impact of land use and urban runoff on the contamination of the Sarno River Basin in Southern Italy. Water Air Sol Pollut 349-366.

7. Blandin, P. (1986). Bioindicateurs et diagnostic des systèmes écologiques. Bull. Ecol 215-307.

8. Bode, R., Novak, M., and Abele, L.. (1991). Quality assurance work plan for biological stream monitoring in New York State. NYS Department of Environmental.

9. Bzioui, M. (2004). Rapport national sur les ressources en eau au Maroc.

10. Echihabi, L., Foutlane, L., and Bourchich, L. (2015). Problèmes majeurs de pollution et leurs impacts sur les ressources en eau au Maroc.

11. Haut-Commissariat aux Eaux et Fôrets et à la Lutte Contre la Désertification [HCEFLCD]. (2006). Etude d'aménagement du bassin versant de l'oued Beht en amont du barrage El Kansera.

12. Hellawell, J.M. (1986). Biological indicators of freshwater pollution and environnemental management. 546.

13. Hilsenhoff, W. (1988). Rapid field assessment of organic pollution with family level biotic index. J. N. Am. Benthol.Soc. 65-68.

14. Kolkwitz, R., and Marsson, M. (1909). Oekologie der tierischen Saprobien. Int. Rev. Hydrobiologie u. Hydrographie 126-152.

15. Liquete, C., Maes, J., La Notte, A., and Bidoglio, G. (2011). Securing water as a resource for society: an ecosystem services perspective. Ecohydrol. Hydrobiol. 11, 247-259. 
16. Malmqvist, B., and Rundle, S. (2002). Threats to the running water ecosystems of the world. Environ. Conserv. 29.

17. Mint Chevie, M. (2011). Contribution à l'étude hydroclimatique du bassin versant de l'oued Beht, Maroc septentrional.

18. Sahili, and Makhokhi (1987). Essais de modélisation de la retenue El Kansera. Eau et Développement 89-105.

19. United Nations Environment Programme Global Environment Monitoring System/ Water Programme [UNEP (GEMS)/WP]. (2008). Water quality for ecosystem and human health.

20. United States Environmental Protection Agency [U.S.EPA]. (1997). Field and laboratory methods for Macroinvertabrates and Habitat Assessement of Low Gradient National Streams, Mid-Atlantic Costal Streams Workgroup Environmental services division, region 3, Wheeling, WV. 